\title{
Ligating clip migration after robot-assisted laparoscopic radical prostatectomy: a single-center experience
}

\author{
Shibin Zhu, Huajian Ye, Haiyang Wu, Guoqing Ding, Gonghui Li^ \\ Department of Urology, Sir Run Run Shaw Hospital, Zhejiang University School of Medicine, Hangzhou, China \\ Contributions: (I) Conception and design: G Li, S Zhu; (II) Administrative support: G Li; (III) Provision of study materials or patients: S Zhu, G Ding; \\ (IV) Collection and assembly of data: H Wu; (V) Data analysis and interpretation: S Zhu; (VI) Manuscript writing: All authors; (VII) Final approval \\ of manuscript: All authors. \\ Correspondence to: Gonghui Li. Department of Urology, Sir Run Run Shaw Hospital, Zhejiang University School of Medicine, No. 3 East Qingchun \\ Road, Hangzhou, 310016, China. Email: 3193119@zju.edu.cn.
}

Background: Ligating clip migration (LCM) after robot-assisted laparoscopic radical prostatectomy (RARP) is a rare but troublesome complication, that may result in calculus formation, bladder neck contracture, and anastomotic stricture. Herein, we describe our experiences with LCM after RARP and explore its risk factors, potential pathogenesis, and preventive measures.

Methods: We retrospectively reviewed patients who underwent RARP at our medical center between December 2015 and June 2019, identifying individuals with LCM. Clinical and surgical data were collected from these patients.

Results: Of the 682 patients who underwent RARP at our institution, 26 (3.8\%) had LCM. The duration from RARP to the identification of LCM ranged from 1 to $37(13 \pm 10)$ months. Clips migrated into the urethrovesical anastomosis in 22 patients (84\%), prompting cytoscopic extraction to remove the migrated clips. The length of stay after RARP was longer in LCM-positive patients than in LCM-negative patients (13.5 vs. 9.4 days, $\mathrm{P}=0.034)$. Additionally, the rates of urine leakage (15\% vs. $6 \%, \mathrm{P}=0.046)$ and anastomotic stenosis $(54 \%$ vs. $5 \%, \mathrm{P}=0.000)$ were higher among $\mathrm{LCM}$-positive patients. More positive urethra/apex margins were found in LCM-positive patients ( $38 \%$ vs. $21 \%, \mathrm{P}=0.039)$.

Conclusions: The incidence of clip migration after RARP may not be as low as previously thought. Cystoscopy is recommended in post-RARP patients with recurrent lower urinary tract symptoms (LUTS) and/or urinary retention. Prolonged length of stay after the first RARP, urine leakage, anastomotic stenosis, and positive urethra/apex margin might be predictors of LCM. We recommend reduced ligating clip usage and electrotome near the urethrovesical anastomosis to reduce clip migration incidence. Meanwhile, more researches are needed to determine the practicality of reducing the risk of clip migration after RARP.

Keywords: Prostatic neoplasms; prostatectomy; ligation

Submitted Jan 02, 2021. Accepted for publication May 21, 2021.

doi: $10.21037 /$ tcr-21-7

View this article at: https://dx.doi.org/10.21037/tcr-21-7

$\wedge$ ORCID: 0000-0001-9845-7241. 


\section{Introduction}

Prostate carcinoma is a common disease that is increasingly being diagnosed. In response, robot-assisted laparoscopic radical prostatectomy (RARP) is being applied to a growing number of patients with localized prostate carcinoma owing to its efficiency and simplicity (1). In this procedure, ligating clips are non-thermal polymer-locking ligation tools that are frequently used in RARP and have been proven to be safe and effective. However, some complications, including hemorrhage and migration, have been reported in few studies (2-4). Moreover, ligating clips may migrate into the adjacent viscera, such as the bladder and urethra, which may result in calculus formation, bladder neck contracture, and anastomotic stricture. Recently, we encountered a few cases with complications of ligating clip migration (LCM) at our medical center. Thus, the purpose of this study was to describe our own experience of clip migration after RARP and to discuss the risk factors, potential pathogenesis, and possible preventive measures. We present the following article in accordance with the STROBE reporting checklist (available at https://dx.doi.org/10.21037/tcr-21-7).

\section{Methods}

The implementation of our robot-assisted surgical system began in December 2015. Thereafter, we retrospectively collected data from 682 patients who underwent RARP between December 2015 and June 2019. RARP was performed by three surgeons, each of whom had an experience of more than 200 cases of regular laparoscopic radical prostatectomy. The patients defined as LCM-positive were confirmed to have undergone RARP and subsequently presented to our outpatient clinic with lower urinary tract symptoms (LUTS) or urinary retention, and they were finally diagnosed with clip migration using cystoscopy. Otherwise, patients were defined as LCM-negative.

The study was conducted in accordance with the Declaration of Helsinki (as revised in 2013) and was approved by the Ethics Committee of Sir Run Run Shaw Hospital, School of Medicine, Zhejiang University (IRB number: 20160222-20). Written informed consent was obtained from all the patients.

\section{Statistical analysis}

Clinical and surgical characteristics were compared across the two patient cohorts using chi-square analysis for categorical variables and $t$-test for continuous variables, with statistical significance set at $\mathrm{P}<0.05$.

\section{Results}

\section{Clinical characteristics of patients underwent RARP}

From December 2015 to June 2019, 682 patients underwent RARP at our hospital. Of these 682 patients, there were 678 prostate carcinoma cases, three benign prostate hyperplasia cases, and one prostate atypical hyperplasia case. Twentysix patients (3.8\%) subsequently presented to our outpatient clinic with LUTS and/or urinary retention and were diagnosed with LCM, requiring endoscopic treatment. The clinical characteristics of the 682 patients are shown in Table 1 .

The mean age was similar in the group of $26 \mathrm{LCM}$ patients and in the group of 656 patients without LCM. The body mass index (BMI) was higher in LCM patients, but the difference was not statistically significant (24.7 vs. $23.7 \mathrm{~kg} / \mathrm{m}^{2}, \mathrm{P}=0.095$ ). Additionally, there were no significant differences in the Gleason grade, preoperative prostatespecific antigen (PSA) level, pathological T stage, status of pelvic lymphadenectomy, or accompanying diseases between the two groups.

\section{LCM after RARP}

Twenty-six patients (3.8\%) after RARP were diagnosed with LCM, wherein the duration from RARP surgery to LCM identification ranged from 1 to $37(13 \pm 10)$ months. Of the 26 patients, ligating clips migrated into the urethrovesical anastomosis in $22(84 \%)$ patients, into the urethra in $2(8 \%)$ patients, and into the bladder cavity in the remaining $2(8 \%)$ (Figure 1). Moreover, stone formation on the ligating clips was observed in 19 (73\%) patients (Figure 1). All 26 patients subsequently underwent cystoscopic removal of the foreign body and/or lithotripsy. Following RARP, two patients had urethral dilation due to an anastomotic stricture before LCM identification. Six (23\%) patients who had their migrated ligating clips removed underwent secondary urethral dilation due to subsequent anastomotic stricture, and one patient underwent two more endoscopic treatments due to subsequent LCM recurrences. The remaining patients recovered well.

\section{Surgical characteristics of patients who underwent RARP}

The distribution of surgical data based on LCM status 
Table 1 Distribution of clinical characteristics in patients underwent robotic-assisted laparoscopic prostatectomy according to LCM status*

\begin{tabular}{|c|c|c|c|c|}
\hline Characteristics & Study population $(\mathrm{n}=682)$ & $\operatorname{LCM}(n=26)$ & No LCM (n=656) & $P$ value \\
\hline BMI & $23.8 \pm 2.9$ & $24.7 \pm 2.7$ & $23.7 \pm 2.9$ & 0.095 \\
\hline Diabetes, $\mathrm{n}[\%]$ & $91[13]$ & $4[15]$ & 87 [13] & 0.767 \\
\hline Hypertension, n [\%] & $326[47]$ & $11[42]$ & $315[48]$ & 0.568 \\
\hline Preoperative PSA, n/total n [\%] & & & & 0.506 \\
\hline$<10 \mathrm{ng} / \mathrm{mL}$ & 225/674 [33] & $11 / 26[42]$ & $214 / 648$ [33] & \\
\hline $10-20 \mathrm{ng} / \mathrm{mL}$ & $218 / 674$ [32] & $6 / 26[[23]$ & 212/648 [33] & \\
\hline$>20 \mathrm{ng} / \mathrm{mL}$ & $231 / 674[34]$ & $9 / 26[35]$ & 222/648 [34] & \\
\hline 7 & $311 / 678[46]$ & $15 / 26[58]$ & $296 / 652[45]$ & \\
\hline$>7$ & $249 / 678$ [37] & $6 / 26[23]$ & $243 / 652[38]$ & \\
\hline T stage, n/total n [\%] & & & & 0.547 \\
\hline $\mathrm{pT} 2 \mathrm{a} / \mathrm{b}$ & $111 / 677[16]$ & $4 / 26[15]$ & $107 / 651[16]$ & \\
\hline pT2c & $306 / 677$ [45] & $9 / 26$ [35] & $297 / 651[46]$ & \\
\hline рТ3a & $113 / 677[17]$ & $7 / 26[27]$ & $106 / 651[16]$ & \\
\hline pT3b & $138 / 677[20]$ & 5/26 [19] & $133 / 651[20]$ & \\
\hline pT4 & 9/677 [2] & $1 / 26[4]$ & 8/651 [2] & \\
\hline
\end{tabular}

*, plus-minus values are means \pm SD. LCM, ligating clip migration; BMI, body weight index; TURP, transurethral resection of prostate; PSA, prostate-specific antigen.

is shown in Table 2. There were no marked or significant differences between LCM-positive and LCM-negative patients in terms of estimated blood loss and surgical time. Contrastingly, the length of stay after RARP was longer in LCM-positive patients (13.5 days) than in LCM-negative patients (9.4 days), which was a small but significant difference $(\mathrm{P}=0.034)$. Urine leakage and anastomotic stenosis rates were also higher among LCMpositive patients (15\% and $54 \%$, respectively) than among LCM-negative patients ( $6 \%$ and $5 \%$, respectively), with statistically significant differences $(\mathrm{P}=0.046$ and $\mathrm{P}=0.000$, respectively). Furthermore, a more positive urethra/apex margin was found in LCM-positive patients than in LCMnegative patients ( $38 \%$ vs. $21 \%, \mathrm{P}=0.039)$.

\section{Discussion}

RARP is a commonly performed minimally invasive procedure for patients with localized prostate carcinoma in our hospital, in which several methods have been used to achieve adequate hemostasis. For this purpose, the ligating clip, known as Hem-o-lok ${ }^{\circledR}$, is a non-thermal ligation equipment extensively used to ligate the prostatic pedicles in RARP.

LCM is found to be a rare complication following laparoscopic radical prostatectomy (2-5), although its overall incidence remains unknown. Herein, we report the greatest number of LCM cases with an incidence of post-RARP clip migration of $3.8 \%$, a number not previously reported by other researchers. In this study, the definite identification time for LCM varied from 1 month to 3 years. Similar to other reports (4-7), nearly all post-RARP patients with clip migration had a history of recurrent LUTS and/or urinary retention. Additionally, most migrated clips had external calcification or stone formation, which may aggravate the previously mentioned symptoms. Therefore, we believe 


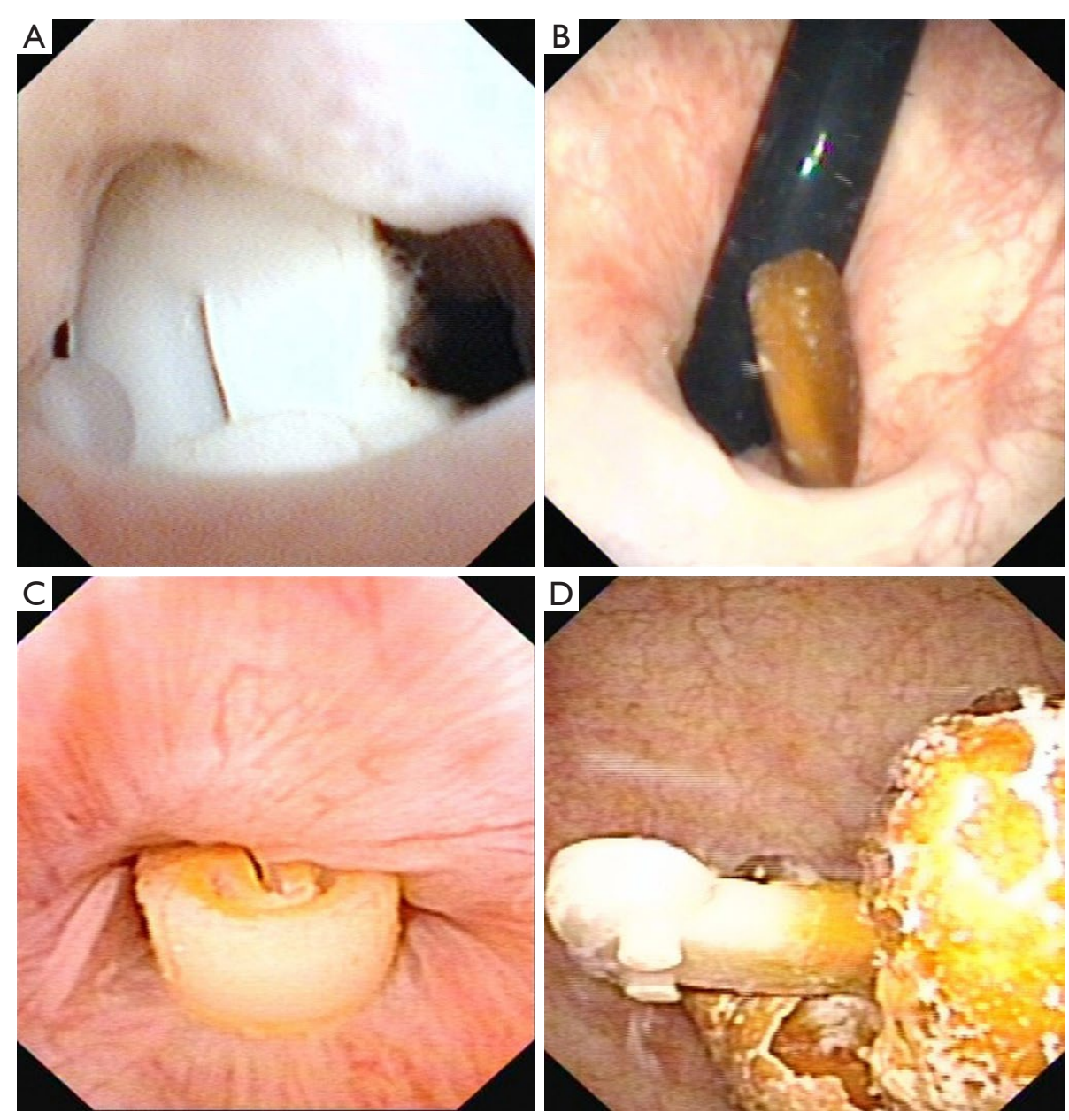

Figure 1 Flexible cystoscopic image showing different positions of clip migration. (A) Clip migration into urethrovesical anastomosis; (B) clip migration into urethrovesical anastomosis with external calcification/stone formation; (C) clip migration into urethra. (D) clip migration into bladder cavity exhibiting as a bladder stone.

Table 2 Characteristics of surgery-related data in patients underwent robotic-assisted laparoscopic prostatectomy according to LCM status*

\begin{tabular}{|c|c|c|c|c|}
\hline Surgery-related data & Study population ( $\mathrm{n}=682$ ) & $\operatorname{LCM}(n=26)$ & No LCM $(n=656)$ & $P$ value \\
\hline Estimated blood loss (mL) & $102 \pm 129$ & $124 \pm 202$ & $101 \pm 126$ & 0.560 \\
\hline Surgical time (minute) & $174 \pm 65$ & $203 \pm 90$ & $172 \pm 64$ & 0.103 \\
\hline \multicolumn{5}{|l|}{ Positive surgical margin, n/total n [\%] } \\
\hline Urethra/apex & $149 / 677$ [22] & $10 / 26[38]$ & $139 / 651[21]$ & 0.039 \\
\hline Pelvic lymphadenectomy, n [\%] & $29[4]$ & $2[8]$ & $27[4]$ & 0.304 \\
\hline Extended lymphadenectomy, n [\%] & $4[1]$ & $0[0]$ & $4[1]$ & 1 \\
\hline Urine leakage, n [\%] & $42[6]$ & $4[15]$ & $38[6]$ & 0.046 \\
\hline Anastomotic stenosis, $\mathrm{n}$ [\%] & $48[7]$ & $14[54]$ & $34[5]$ & 0.000 \\
\hline
\end{tabular}

*, plus-minus values are means \pm SD. LCM, ligating clip migration; LOS, length of stays; RLRP, robot-assisted laparoscopic radical prostatectomy. 
that clip migration should be considered in patients with recurrent LUTS and/or urinary retention, even if over a long period has passed after RARP.

Based on our experience, cystoscopy is recommended for LCM identification since cystoscopic removal of foreign bodies and/or lithotripsy is the preferred alternative for clip migration with or without external calcification. In our study, most patients recovered well after cystoscopic treatment, while urethral dilation was still necessary in patients with anastomotic stenosis. Furthermore, recurrence of clip migration is rare but should not be overlooked.

For clip migration cases, we reviewed most surgical videos and found that the clips were not inside but close to the vesicourethral anastomosis in RARP. We then speculated that post-RARP clip migration may follow from secondary healing of the vesicourethral anastomosis, which can be caused by devitalization, ischemia, infection, and necrosis of the anastomotic tissue, namely the bladder neck and proximal urethra. Poor healing and inflammatory reactions in the second intention process contribute to anastomosis disruption that can result in clip erosion and migration into the anastomosis, causing subsequent migration into the urethra and bladder. Once the clip is in contact with urine, it serves as a nidus for calcification and stone formation.

Moreover, clip migration risk after RARP may vary and be complicated (6-8). Turini et al. (8) reported that increased length of hospital stays and the need for larger blood transfusion volumes following prostatectomy were significant predictors of clip migration. In our study, LCM patients had longer average hospital stays and higher urine leakage rates following RARP, with most of them not recovering well after their first RARP surgery. Some of them even had one or more complications, such as fever, surgical site pain, lymphatic leakage, or urine leakage, which resulted in a longer hospital stay. In contrast, surgical time, estimated blood loss, preoperative Gleason grade, preoperative PSA level, and pathological T stage did not serve as predictive factors for clip migration.

Due to the imaging system's high resolution, a mucosato-mucosa vesicourethral anastomosis can be achieved in most RARP cases. There were a few patients who developed urine leakage (6\%) and anastomotic stenosis (7\%), wherein this proportion was higher among LCM-positive patients (15\% and $54 \%$, respectively). We believe that poor healing and/or secondary healing might have resulted in postoperative urine leakage and subsequent clip migration given that anastomotic stenosis is mainly caused by scarring and vesicourethral anastomosis contraction. In our study,
$54 \%$ of LCM patients were accompanied with anastomotic stenosis, indicating that poor healing and/or secondary vesicourethral anastomosis healing could potentially be causes of clip migration and anastomotic stenosis; however, these results do not prove causality.

Our study also indicates that a positive urethra/apex margin might be relevant to subsequent clip migration after RARP. A higher rate of positive urethra/apex margin might indicate more difficulty in separating the apex and urethra due to fragile tissue or severe adhesion. Thus, more electrotome energy and clips were used to stop the bleeding around the apex, leaving the vesicourethral anastomosis more likely to become ischemic and necrotic. Therefore, clips were more likely to migrate due to the poor anastomotic healing.

In this study, cold scissors were used to separate the prostate apex and urethra during regular laparoscopic radical prostatectomy. However, more electrome energy and clips were used to achieve hemostasis due to the enlarged bleeding effect under robotic vision. This may cause ischemia and anastomotic tissue necrosis, which leads to a high risk of clip migration. To prevent clip migration after RARP, we reduced the number of ligating clips to four to six clips per case, and these were only used at the proximal part of the prostatic pedicles (Figure 2). For the distal end of the prostatic pedicles, suture ligation and minimum electrocoagulation are alternative methods to ligating clips for achieving hemostasis, wherein continuous sutures are used to control bleeding along the neurovascular bundle. Every attempt was made to minimize ligating clips using nearby vesicourethral anastomosis (Figure 2). Electrotome use during the separation between the prostate apex and urethra may cause anastomotic tissue devitalization and necrosis, thus we now used scissors to separate them instead. After we reduced electrotome usage and clips near the prostate apex and urethra, clip migration cases were hardly seen in the last 2 years. Given the delay in post-RARP LCM presentation of up to 3 years, more observation is needed to determine if this change in surgical technique will be effective in reducing clip migration.

\section{Conclusions}

In summary, the LCM incidence after RARP might not be as rare as we have initially considered. Cystoscopy is recommended in post-RARP patients with recurrent LUTS and/or urinary retention. Prolonged length of stay after the first RARP, urine leakage, anastomotic stenosis, 

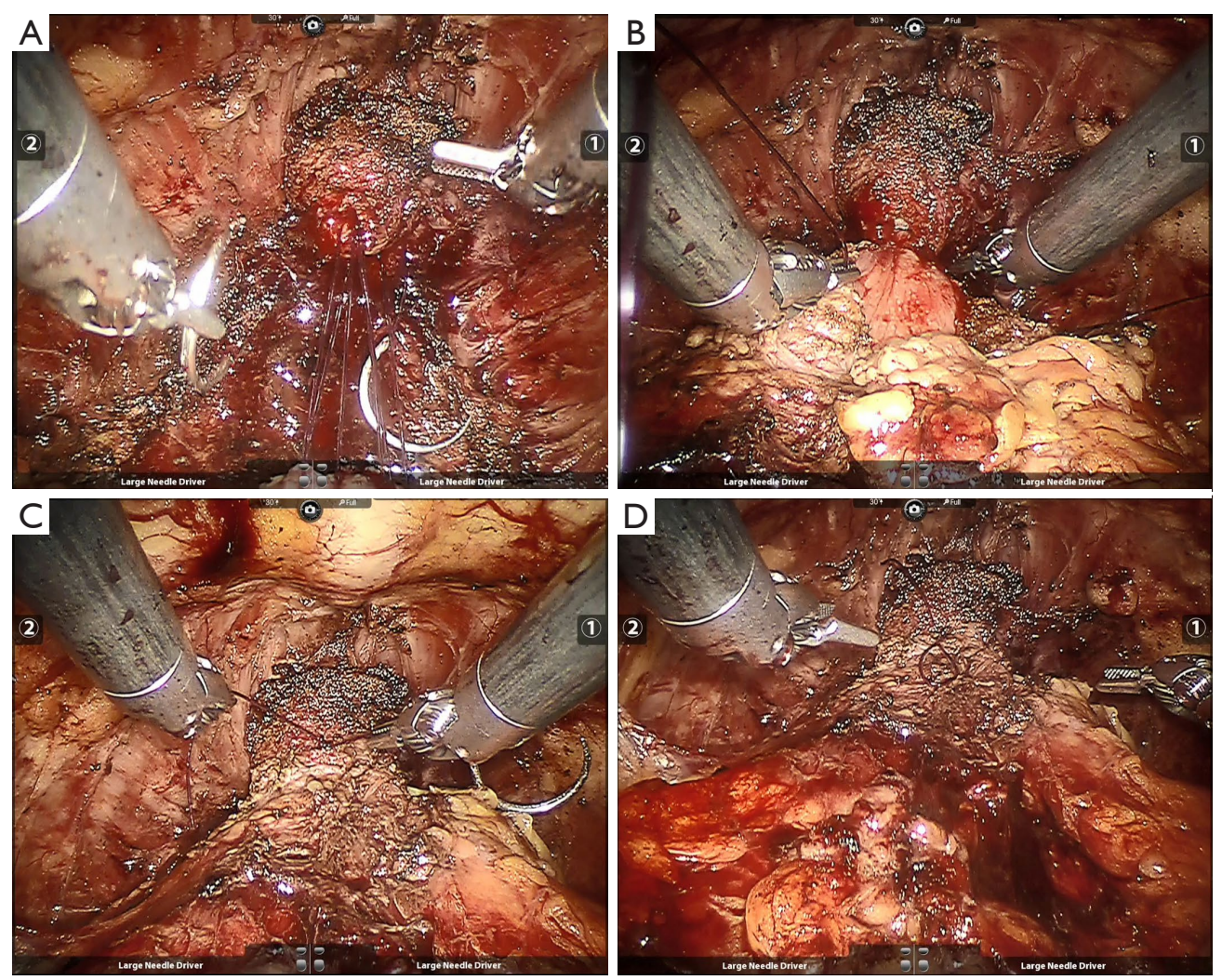

Figure 2 Reduced clips and electrotome usage near the urethrovesical anastomosis. (A) Reduced clip usage and less electrotome energy; (B) mucosa-to-mucosa anastomosis; (C) continuous suture; (D) final status.

and positive urethra/apex margin might be predictors of LCM. Furthermore, we suggested reduced ligating clip and electrotome usage near the urethrovesical anastomosis; however, more research is needed to determine the practicality of these measures in reducing the risk of clip migration after RARP.

\section{Acknowledgments}

We acknowledge Language Service of Zhejiang University Press (Hangzhou, China) for English editing.

Funding: This work was supported by Medical Health Science and Technology Project of Zhejiang Provincial Health Commission (2018KY470); Natural Science Foundation of Zhejiang Province (LQ19H050011).

\section{Footnote}

Reporting Checklist: The authors have completed the STROBE reporting checklist. Available at https://dx.doi. org/10.21037/tcr-21-7

Data Sharing Statement: Available at https://dx.doi. org/10.21037/tcr-21-7

Conflicts of Interest: All authors have completed the ICMJE uniform disclosure form (available at https://dx.doi. org/10.21037/tcr-21-7). The authors have no conflicts of interest to declare.

Ethics Statement: The authors are accountable for all aspects of the work in ensuring that questions related to the accuracy or integrity of any part of the work are appropriately investigated and resolved. The study was conducted in accordance with the Declaration of Helsinki (as revised in 2013). The present study was approved by the Ethics Committee of Sir Run Run Shaw Hospital, School of Medicine, Zhejiang University (IRB number: 2016022220). Written informed consent was obtained from all the patients. 
Open Access Statement: This is an Open Access article distributed in accordance with the Creative Commons Attribution-NonCommercial-NoDerivs 4.0 International License (CC BY-NC-ND 4.0), which permits the noncommercial replication and distribution of the article with the strict proviso that no changes or edits are made and the original work is properly cited (including links to both the formal publication through the relevant DOI and the license). See: https://creativecommons.org/licenses/by-nc-nd/4.0/.

\section{References}

1. Wallerstedt A, Tyritzis SI, Thorsteinsdottir T, et al. Shortterm results after robot-assisted laparoscopic radical prostatectomy compared to open radical prostatectomy. Eur Urol 2015;67:660-70.

2. Banks EB, Ramani A, Monga M. Intravesical Weck clip migration after laparoscopic radical prostatectomy. Urology 2008;71:351.e3-4.

3. Blumenthal KB, Sutherland DE, Wagner KR, et al. Bladder neck contractures related to the use of Hem-o-lok clips in robot-assisted laparoscopic radical prostatectomy.

Cite this article as: Zhu S, Ye H, Wu H, Ding G, Li G. Ligating clip migration after robot-assisted laparoscopic radical prostatectomy: a single-center experience. Transl Cancer Res 2021;10(7):3429-3435. doi: 10.21037/tcr-21-7
Urology 2008;72:158-61.

4. Mora ER, Galí OB, Garin JA, et al. Intravesical migration and spontaneous expulsion of a Hem-o-lok polymer ligating clip after laparoscopic radical prostatectomy. Urology 2010;75:1317.

5. Cormio L, Massenio P, Lucarelli G, et al. Hem-o-lok clip: a neglected cause of severe bladder neck contracture and consequent urinary incontinence after robot-assisted laparoscopic radical prostatectomy. BMC Urol 2014;14:21.

6. Bientinesi R, Di Gianfrancesco L, Pugliese D, et al. Endourethral migration of a Hem-o-Lok Clip after robotassisted laparoscopic radical prostatectomy. Urologia 2015;82:242-4.

7. Yu CC, Yang CK, Ou YC. Three types of intravesical Hem-o-Lok clip migration after laparoscopic radical prostatectomy. J Laparoendosc Adv Surg Tech A 2015;25:1005-8.

8. Turini GA 3rd, Brito JM 3rd, Leone AR, et al. Intravesical hemostatic clip migration after robotic prostatectomy: case series and review of the literature. J Laparoendosc Adv Surg Tech A 2016;26:710-2. 